\title{
Predicting Functional Coronal Knee Laxity in Pre-Operative TKA Patients Using Morphological Measurements
}

\author{
Cerys Edwards $^{1,2}$, Ella M. Moore ${ }^{1,3}$, Willy Theodore ${ }^{1,3}$, Joshua Twiggs ${ }^{1,3}$, \\ Edgar A. Wakelin ${ }^{1}$, David Liu ${ }^{4}$, Brad Miles ${ }^{1,2}$ \\ ${ }^{1} 360$ Knee Systems, Sydney, Australia \\ ${ }^{2}$ University of New South Wales, Sydney, Australia \\ ${ }^{3}$ University of Sydney, Sydney, Australia \\ ${ }^{4}$ Gold Coast Centre for Bone and Joint Surgery, Queensland, Australia \\ joshua@kneesystems.com, brad@_kneesystems.com
}

\begin{abstract}
Currently, pre-operative analysis of soft-tissue balance is limited to measures of passive laxity rather than active laxity. By including active laxity data, a more comprehensive surgical plan can be delivered, however there are no measures for active laxity currently in routine use. Therefore, the validation of a proxy measure based on routine collected imaging is valuable. This study aimed to determine whether coronal knee laxity can be predicted from pre-operative alignment and bony morphology of the knee. Fifty-eight patients with pre-operative $\mathrm{CT}$ and stressed x-ray imaging for activity laxity were analysed to identify anatomical landmarks and determine varus-valgus laxity ranges for a range of flexion angles with the joint subjected to lateral forces. Correlations between anatomical and alignment parameters, vs laxity ranges and midpoints were determined using pairwise complete Pearson linear correlation analyses. Of the 17 anatomical/alignment measurements studied, 8 correlated significantly with the knee laxity range's midpoint at $20^{\circ}$ flexion, with the strongest correlation being with supine coronal alignment $(r=0.95, \mathrm{p}<0.001)$; the findings were similar at $45-90^{\circ}$. Compared to knee laxity midpoint, knee laxity range was not as strongly correlated with anatomical and alignment parameters, with only 3 anatomical parameters correlated significantly with laxity range at $20^{\circ}$ flexion and none at $45-90^{\circ}$ flexion. These results suggest morphological measurements and anatomical characteristics may help define functional coronal laxity range of the knee.
\end{abstract}




\section{Introduction}

One of the primary goals of total knee arthroplasty (TKA) surgery is to establish a soft-tissue friendly laxity profile through the full range of motion [1]. If appropriate ligament balance is not achieved, stiffness or instability of the knee joint can occur [2]. Currently, pre-operative surgical analysis of softtissue balance is limited to examination of static images (X-ray \& CT) [3, 4], combined with intraoperative passive assessment of laxity by subjective force, navigation, tensioners or spacer blocks. [5, 6]. None of these coronal knee laxity assessments establish the patient's active laxity profile, which may be an important consideration for achieving optimal soft-tissue balance during surgery. Active stress X-rays can be captured pre-operatively to define the patient specific coronal laxity, however, these assessments are not routine. The purpose of this study is to determine if active laxity profiles gathered from stress x-rays could be predicted from a patient's pre-operative alignment \& bony morphology. Ascertaining these relationships could allow surgeons to better understand the active preoperative laxity profile prior to surgery - allowing for more comprehensive pre-operative planning \& the establishment of surgical knee laxity goals post. The study hypothesis is that coronal knee laxity can be predicted from pre-operative alignment and bony morphology of the knee.

\section{Methods}

Fifty-eight patients with an average age of 67 were retrospectively analysed. Pre-operative CT scans were captured for each patient and used to generate 3D bone models, from which anatomical landmarks were identified. These landmarks were used to calculate 17 morphological \& alignment measurements (three examples are shown in Figure 1A-C). Stress x-rays were obtained pre-operatively using a Telos (METAX, Germany) stress device to apply varus \& valgus forces at $20^{\circ}$ extension and between $45 \&$ $90^{\circ}$ flexion. The 3D CT-generated bone models were then registered to the $2 \mathrm{D}$ stress $\mathrm{x}$-rays using Mimics (Materialise, Belgium) Research software (Figure 1D). Landmarks acquired from the preoperative $\mathrm{CT}$ scan to define patient specific anatomic axes were then transformed to the stressed bone locations and used to calculate the varus and valgus extents of the patient's laxity range. The laxity midpoint of the knee was also found by taking the average of the varus and valgus extents. Correlations between anatomical landmarks and laxity ranges and midpoints were determined using pairwise complete Pearson linear correlation analyses.

\section{Results}

Of the 17 morphological/alignment measurements included in this study, 47\% (8/17) correlated significantly with knee laxity midpoint at $20^{\circ}$ flexion \& 59\% (10/17) correlated significantly with knee laxity midpoint at $45-90^{\circ}$ flexion. The strongest correlation was between knee laxity midpoint at $20^{\circ}$ flexion and supine coronal alignment $(\mathrm{r}=0.95, \mathrm{p}<0.001)$. Only 18\% (3/17) of anatomical measurements correlated significantly with laxity range at $20^{\circ}$ flexion and no significant correlations were identified with laxity range at $45-90^{\circ}$ flexion. The anatomical measurements that correlated significantly with laxity range at $20^{\circ}$ flexion were coronal angle between the anatomic and mechanical axes of the femur $(\mathrm{r}=-0.34, \mathrm{p}=0.008)$ (Figure 3), external rotation of Whiteside's axis from the transepicondylar axis of the femur $(r=-0.28, p=0.033) \&$ the supine lateral joint gap $(r=-0.31, p=$ $0.018)$. 


\section{Discussion}

The absence of correlation between the flexion laxity range and coronal alignment indicates coronal alignment alone is insufficient to characterise the knee laxity profile. Pre-operative laxity range may be an important measurement for surgeons when determining a target post-operative knee laxity [7]. Obtaining stressed X-rays to determine functional laxity ranges however, is not practical for routine TKA pre-operative analysis and consequently we investigated whether patient specific knee laxity could be predicted from anatomical features on CT scan and radiographs. Previous investigations have found correlations between knee osteoarthritis, alignment and laxity [8], but have not investigated morphological measures on a 3 dimensional patient specific basis. At this stage knee laxity at $20^{\circ}$ flexion has shown a significant correlation, indicating that with greater data, bone morphology may assist in characterising the soft tissue profile. As the dataset builds and further morphological measurements are explored, coronal laxity through a full range of motion may be predictable, but at this stage specialised radiographs or an alternative intra-operative measurement technique is required.

\section{Conclusion}

Morphological measurements and anatomical characteristics may help define functional coronal laxity range of the knee. Alternative methods in addition to static imaging need to be explored to define patient specific coronal knee laxity. These may include functional knee radiographs or intra-operative measurement techniques or both combined.
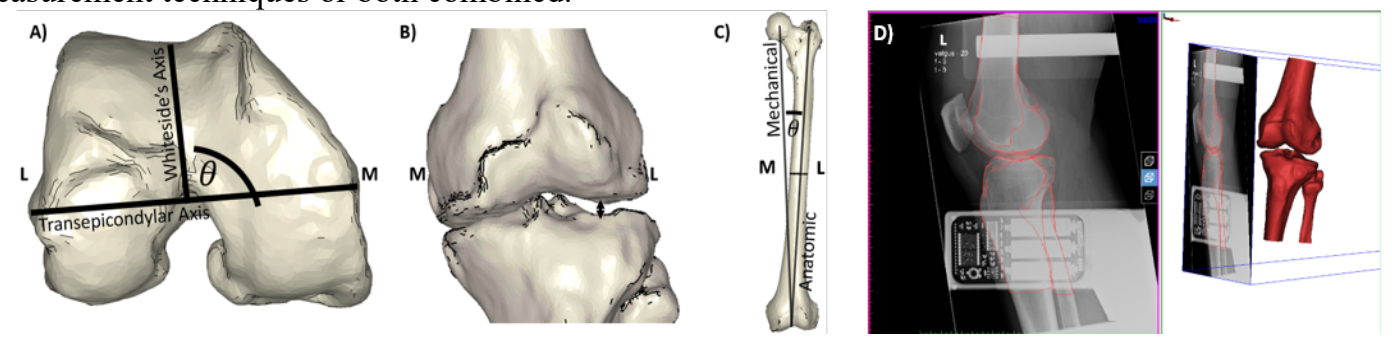

Figure 1: Examples of anatomical measurements included in this analysis. A) External rotation of Whiteside's axis to the transepicondyar axis of the femur. B) Supine lateral joint gap. C) Coronal angle between the mechanical to anatomic axes of the femur. D) Registration of bone models to stressed x-rays in Mimics.

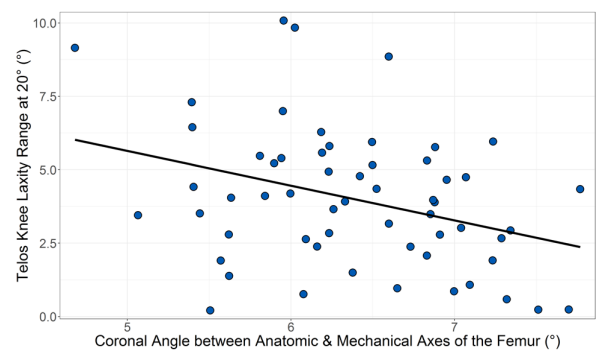

Figure 2: The relationship between the angle between the anatomic \& mechanical axes of the femur \& knee laxity at $20^{\circ}$. 


\section{References}

1 Wilton, T., A. Sambatakakis, and S. Attfield, Soft-tissue balancing at the time of knee replacement: rationale and method. The Knee, 1994. 1(2): p. 111116.

2. Rodriguez-Merchan, E.C., Instability following total knee arthroplasty. HSS journal, 2011. 7(3): p. 273.

3. Stähelin, T., et al., Fluoroscopically assisted stress radiography for varusvalgus stability assessment in flexion after total knee arthroplasty. The Journal of arthroplasty, 2003. 18(4): p. 513-515.

4. Okazaki, K., et al., Asymmetry of mediolateral laxity of the normal knee. Journal of Orthopaedic Science, 2006. 11(3): p. 264.

5. D'Lima, D., et al. Novel electronic intraoperative ligament balance predicts postoperative knee kinematics. in Orthopaedic Proceedings. 2016. The British Editorial Society of Bone \& Joint Surgery.

6. Smith, T., et al., How are we addressing ligament balance in TKA? A literature review of revision etiology and technological advancement. Journal of clinical orthopaedics and trauma, 2016. 7(4): p. 248-255.

7. Roth, J.D. and S.M. Howell, Soft tissue balance of the native knee provides guidance for balancing a total knee arthroplasty, in Soft Tissue Balancing in Total Knee Arthroplasty. 2017, Springer. p. 17-27.

8. Hicks-Little, C.A., et al., The Relationship Between Early-Stage Knee Osteoarthritis and Lower-Extremity Alignment, Joint Laxity, and Subjective Scores of Pain, Stiffness, and Function. Journal of sport rehabilitation, 2016. 25(3): p. 213-218. 